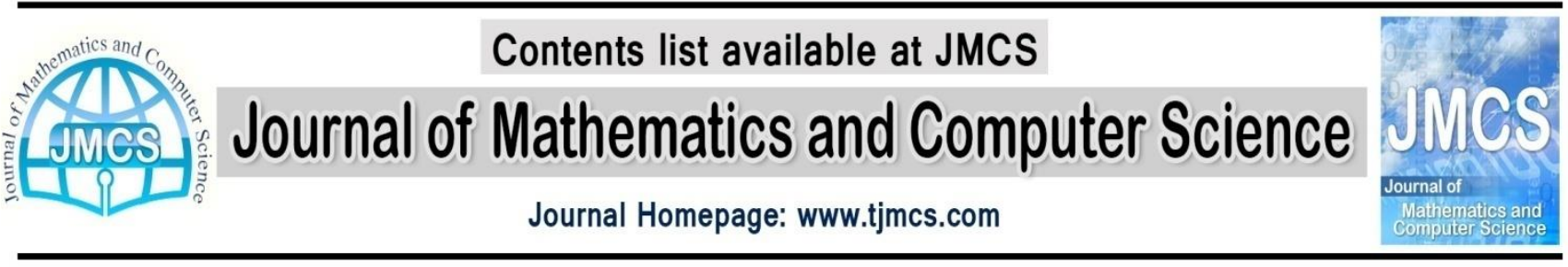

\title{
Rational Laguerre Functions and Their Applications
}

\author{
A. Aminataei1 ${ }^{1,}$, S. Ahmadi-Asl' ${ }^{2}$, Z. KalatehBojdi ${ }^{2}$ \\ ${ }^{1}$ Department of Applied Mathematics, Faculty of Mathematics, K. N. Toosi University of Technology, \\ P.O. Box 16315-1618, Tehran, Iran \\ *ataei@kntu.ac.ir \\ ${ }^{2}$ Department of Mathematics, Birjand University, Birjand, Iran \\ Article history: \\ Received July 2014 \\ Accepted August 2014 \\ Available online November 2014
}

\begin{abstract}
In this work, we introduce a new class of rational basis functions defined on $[a, b)$ and based on mapping the Laguerre polynomials on the bounded domain $[a, b)$. By using these rational functions as basic functions, we implement spectral methods for numerical solutions of operator equations. Also the quadrature formulae and operational matrices (derivative, integral and product) with respect to these basis functions are obtained. We show that using quadrature formulae based on rational Laguerre functions give us very good results for numerical integration of rational functions and also implementing spectral methods based on these basis functions for solving stiff systems of ordinary differential equationsgive us suitable results. The details of the convergence rates of these basis functions for the solutions of operator equations are carried out, both theoretically and computationally and the error analysis is presented in $L^{2}([a, b))$ space norm.
\end{abstract}

Keywords: Rational Laguerre functions, Spectral methods, Quadrature formulae, Stiff system, Hilbert space.

\section{Introduction}

Orthogonal polynomials play a prominent role in pure, applied and computational mathematics, as well as in the applied sciences and also in the many fields of numerical analysis such as quadratures, approximation theory and so on [1-4]. In particular case, these polynomials have an important role in the spectral methods. These methods (spectral methods) have been successfully applied in the approximation of partial, differential and integral equations. Three most widely used spectral versions are the Galerkin, collocation and Tau methods. Their utility is based on the fact that if the solution sought is smooth, usually only a few terms in an expansion of global basis functions are needed to represent it to high accuracy [5-11].

We must note to this point that numerical methods for ordinary, partial and integral differential equations can be classified into the local and global categories. The finite-difference and finite-element methods are based on local arguments, whereas the spectral methods are in a global class [12,13]. Spectral methods, in 
the context of numerical schemes for differential equations, belong to the family of weighted residual methods, which are traditionally regarded as the foundation of many numerical methods such as finite element, spectral, finite volume and boundary element methods. There are essentially three important questions that we have to make to derive a spectral method: i: what expansion functions should be used? ii: the form in which the approximation will be written? And finally iii: the procedure by which the solution unknowns are determined? The first question or selecting the global basis functions for expansion of functions has an important role in implementing spectral methods, and this choice depend on the behavior of an exact solution of the problem. For instance, if an exact solution has periodic behavior, then trigonometric functions are the suitable choices for basic functions [14]. Also in nonperiodic cases the polynomial solutions of eigenvalue problems in ordinary differential equations, known as Sturm-Liouville problems on the interval $[-1,1]$ are suitable choices. The Jacobi polynomials are the well-known class of polynomial solutions of nonperiodic Sturm-Liouville problem exhibiting spectral convergence, of which particular examples are Chebyshev polynomials of the firrst and second kinds, and Legendre polynomials in which have high applications [8-15]. In the numerical solutions of ordinary, partial and integral differential equations on the unbounded domains, also some basic functions are named as rational Jacobi functions are used [16-18]. In this work, we introduce a new set of rational basis functions defined on $[a, b)$ and based on mapping the Laguerre polynomials on the bounded domain $[a, b)$. using these rational functions as basic functions, we implement spectral methods for numerical solution of the operator equations. The importance and superiority of the new basis functions rather than other basis functions such as polynomials are shown for one experiment with stiff solution. The details of the convergence rates of these basis functions for solution of the operator equations are carried out, both theoretically and computationally. This work is organized as follows. In section 2, we introduce the Laguerre polynomials and rational Laguerre functions (generalized) and their properties such as their quadrature formulae and etc. In section 3, we present the derivative, integral and product operational matrices with respect to rational Laguerre functions. Section 4, is devoted to the approximation with rational Laguerre functions and convergence rates of these basis functions for solutions of the operator equations. One experiment is presented in section 5, for showing the accuracy of our development. Finally in section 6, we have monitored a brief conclusion.

\section{The Laguerre polynomials (generalized)}

In this part, we define the Laguerre polynomials (generalized) and their properties such as their SturmLiouville ordinary differential equation, three terms recursion formula and etc. Let $\Lambda=(0,+\infty)$, then Laguerre polynomials are denoted by $L_{n}^{\alpha}(x)(\alpha>-1)$, and they are the Eigen functions of the Sturm Liouville problem

(1)

$$
x^{-\alpha} e^{x}\left(x^{\alpha+1} e^{-x}\left(L_{n}^{\alpha}(x)\right)^{\prime}\right)^{\prime}+\lambda_{n} L_{n}^{\alpha}(x)=0, x \in \Lambda
$$

with the eigenvalues $\lambda_{n}=n[12,13]$.

Laguerre polynomials are orthogonal in $L_{w^{\alpha}}^{2}(\Lambda)$ space with the weight function $w_{\alpha}(x)=x^{\alpha} e^{-x}$, satisfy in the following relation

$$
\int_{0}^{+\infty} L_{n}^{\alpha}(x) L_{m}^{\alpha}(x) w_{\alpha}(x) d x=\gamma_{n}^{\alpha} \delta_{m, n}, \quad \gamma_{n}^{\alpha}=\frac{\Gamma(n+\alpha+1)}{\Gamma(n+1)},
$$

where $\delta_{m, n}$ is kronecker delta function. The explicit form of these polynomials is in the form 


$$
L_{n}^{\alpha}(x)=\sum_{i=0}^{n} E_{i}^{\alpha} x^{i}
$$

where

$$
E_{i}^{\alpha}=\frac{\left(\begin{array}{c}
n+\alpha \\
n-i
\end{array}\right)(-1)^{i}}{i !} .
$$

These polynomials are satisfied in the following three terms recurrence formula

$$
\begin{gathered}
(n+1) L_{n+1}^{\alpha}(x)=(2 n+\alpha+1-x) L_{n}^{\alpha}(x)-(n+\alpha) L_{n-1}^{\alpha}(x), \\
L_{0}^{\alpha}(x)=1, L_{1}^{\alpha}(x)=\alpha+1-x .
\end{gathered}
$$

The case $\alpha=0$, leads to the classical Laguerre polynomials, which are used most frequently in practice and will simply be denoted by $L_{n}(\mathrm{x})$. An important property of the Laguerre polynomials is the following derivative relation $[12,13]$ :

$$
\left(L_{n}^{\alpha}(x)\right)^{\prime}=\sum_{i=0}^{n-1} L_{i}^{\alpha}(x)
$$

Further, $\left(L_{i}^{\alpha}(x)\right)^{(k)}$ are orthogonal with respect to the weight function $w_{\alpha+k}$. i.e.

$$
\int_{0}^{+\infty}\left(L_{i}^{\alpha}\right)^{(k)}(x)\left(L_{j}^{\alpha}\right)^{(k)}(x) w_{\alpha+k}(x) d x=\gamma_{n-k}^{\alpha+k} \delta_{i, j},
$$

where $\gamma_{n-k}^{\alpha+k}$ is defined in (2).

\subsection{The rational Laguerre functions (generalized)}

In this section, we present the rational Laguerre functions (generalized) as orthogonal basis functions. At first we use a suitable change of variable formula, in which transform the un bounded domain $(0, \infty)$ (domain of Laguerre polynomials) to the bounded domain $[a, b)$. Using this change of variable formula and substituting it in the Laguerre polynomials, we define a new set of rational orthogonal basis functions in the bounded domain. For this purpose, let $\phi(x, h)$ be a strictly increasing function with respect to a positive parameter $h$; or

$$
\begin{gathered}
y=\phi(x, h), h>0, \quad x \in[a, b), \\
y \in(0,+\infty) .
\end{gathered}
$$

However the condition $\phi^{\prime}(x, h)>0$, is equivalent to the strictly increasing property of $\phi(x, h)$.

Several types of transformation $\phi(x, h)$ are exist for mapping the unbounded domain to bounded domain such as: 


$$
y=\frac{h(x-a)}{b-x}, x=\frac{b y+h a}{y+h},
$$

in which transform between $x \in[a, b)$ and $y \in(0, \infty)$.

This transformation (10) is named as an algebraic transformation. Now using the change of variable $\frac{h(x-a)}{b-x}$ (algebraic transformation), we construct a new set of orthonormal basis functions in a particular weighted Sobolev $\quad$ spaces $($ on the interval $[a, b) \quad$ ). Let

$$
R_{i}^{\alpha}(x, h)=L_{i}^{\alpha}\left(\frac{h(x-a)}{b-x}\right), x \in[a, b),
$$

then $R_{i}^{\alpha}(x, h)$ are the eigenfunctions of the Sturm-Liouville problem

$$
\frac{(x+h)^{2}}{h(b-a)} x^{-\alpha} e^{x}\left(\frac{(x+h)^{2}}{h(b-a)} x^{\alpha+1} e^{-x}\left(R_{i}^{\alpha}(x, h)\right)^{\prime}\right)^{\prime}+\lambda_{n} R_{i}^{\alpha}(x, h)=0,
$$

with the corresponding eigenvalues $\lambda_{n}=n$. We call $R_{i}^{\alpha}(x, h)$ as the rational Laguerre functions and the orthogonality relation for these functions is

$$
\int_{-1}^{1} R_{m}^{\alpha}(x, h) R_{n}^{\alpha}(x, h) w_{r}^{\alpha}(x) d x=\gamma_{n}^{\alpha} \delta_{m, n},
$$

where

$$
w_{r}^{\alpha}(x)=\left(\frac{h(x-a)}{b-x}\right)^{\alpha} e^{-\frac{h(a+x)}{b-x}} k(x),
$$

$k(x)=\left(\frac{h(x-a)}{(b-x)}\right)^{\prime}, \gamma_{n}^{\alpha}$ is defined in (2) and $\delta_{m, n}$ is a kronecker delta function. Moreover, the recurrence relations (5) and (6), imply that

$$
\begin{gathered}
(n+1) R_{n+1}^{\alpha}(x, h)=\left(2 n+\alpha+1-\frac{h(x-a)}{b-x}\right) R_{n}^{\alpha}(x, h)-(n+\alpha) R_{n-1}^{\alpha}(x, h), \\
R_{0}^{\alpha}(x, h)=1, R_{1}^{\alpha}(x, h)=\alpha+1-\frac{h(x-a)}{b-x}, \\
\left(\frac{(x+h)^{2}}{h(b-a)}\right)\left(R_{n}^{\alpha}(x, h)\right)=\sum_{i=0}^{n-1} R_{i}^{\alpha}(x, h) .
\end{gathered}
$$

Also let us consider the following notation

$$
R_{n}^{h}=\operatorname{span}\left\{L_{i}^{\alpha}\left(\frac{h(x-a)}{b-x}\right), i=0, \ldots, n\right\},
$$

in which is needed in this work later.

\subsection{The quadrature formulae with respect to rational Laguerre functions (generalized)}

In this part, we introduce the Gauss and Gauss-Radau quadrature formulae based on rational Laguerre functions. For this purpose, let us first consider the following theorem.

Theorem 1.Let $x_{j}^{(\alpha)}, w_{j}^{(\alpha)}$ be the nodes and weights associated with the Gauss or Gauss-Radauquadratures for Laguerre polynomials. Then 


$$
\int_{0}^{+\infty} f(x) w^{\alpha}(x) d x=\sum_{j=0}^{n} f\left(x_{j}^{(\alpha)}\right) w_{j}^{(\alpha)}+\mathrm{R}_{n, \delta}(x)
$$

where

$$
\mathrm{R}_{n, \delta}(x)=\frac{f^{(2 n+\delta)}(\xi) \gamma_{n}^{\alpha}}{2 n+\delta},
$$

Further $\delta=0$ and 1, for Gauss and Gauss-Radauquadratures respectively, and for some $\xi \in[0,+\infty)$. For the Gauss quadrature case, $\left\{x_{j}^{(\alpha)}\right\}_{j=1}^{n}$ are the zeros of $L_{n}^{\alpha}(x)$ and

$$
\begin{aligned}
w_{j}^{(\alpha)}=-\frac{\Gamma(n+\alpha+1)}{(n+1) !} \frac{1}{L_{n}^{(\alpha)}\left(x_{j}^{(\alpha)}\right)\left(L_{n+1}^{(\alpha)}\right)^{\prime}\left(x_{j}^{(\alpha)}\right)} \\
=\frac{\Gamma(n+\alpha+1)}{(n+\alpha+1)(n+1) !\left(L_{n}^{(\alpha)}\left(x_{j}^{(\alpha)}\right)\right)^{2}}, 0 \leq j \leq n,
\end{aligned}
$$

and for the Gauss-Radau quadrature, $\left\{x_{j}^{(\alpha)}\right\}_{j=1}^{n}$ are the zeros of $\left(L_{n+1}^{(\alpha)}(x)\right)^{\prime}$ and

$$
\begin{gathered}
w_{0}^{(\alpha)}=\frac{(\alpha+1) \Gamma^{2}(\alpha+1) \Gamma(N+1)}{\Gamma(N+\alpha+1)}, \\
w_{j}^{(\alpha)}=\frac{\Gamma(N+\alpha+1)}{N ! \Gamma(N+\alpha+1)} \frac{1}{\left[\left(L_{N}^{(\alpha)}\right)^{\prime}\left(x_{j}^{(\alpha)}\right)\right]^{2}}=\frac{\Gamma(N+\alpha+1)}{N ! \Gamma(N+\alpha+1)} \frac{1}{\left[L_{N}^{(\alpha)}\left(x_{j}^{(\alpha)}\right)\right]^{2}}, 1 \leq j \leq n,
\end{gathered}
$$

Proof: see [13].

Now from (17), we can obtain the following result:

Let $x_{j}^{(\alpha)}, w_{j}^{(\alpha)}$ be the nodes and weights associated with the Gauss or Gauss-Radau quadrature for Laguerre polynomials. Then

$$
\int_{a}^{b} f(x) w_{r}^{\alpha}(x) d x=\sum_{j=0}^{n} f\left(y_{j}^{(\alpha)}\right) w_{j}^{(\alpha)}+\mathrm{R}_{n, \delta}^{h}(x)
$$

where

$$
\mathrm{R}_{n, \delta}^{h}(x)=\frac{\left.\left(f\left(\frac{h(x-a)}{b-x}\right)\right)^{(2 n+\delta)}\right|_{x=\varsigma} \gamma_{n}^{\alpha}}{(2 n+\delta)}
$$

and

$$
y_{j}^{(\alpha)}=\frac{h\left(x_{j}^{(\alpha)}-a\right)}{b-x_{j}^{(\alpha)}} .
$$

Further, $\delta=1$, and 0 ; for Gauss and Gauss-Radau quadrature respectively, and for some $\varsigma \in[0,+\infty)$.

Remark 1.As we see from (17), we can prove that the integration formula (21) is exact for all $p \in R_{2 n+\delta}^{h}$ where $R_{2 n+\delta}^{h}$ is defined in (16). However it is expected that for rational functions, using quadrature (21), we obtain better results than other polynomial base methods, because of the similar behavior of a function and its basis functions for approximation. Now for showing this fact and the accuracy of quadrature formula (21), we present some experiments. 
Experiment 1.Consider the following integral

$$
\int_{0}^{0.5} \frac{x^{2}-2 x-3}{(x-1)\left(x^{2}+2 x+2\right)} d x
$$

then the obtained results from rational Laguerre gauss (RLG) and Legendre gauss (LG) methods are shown in table 1.

Table 1: Comparison between RLG and LG methods for computation of (24) of experiment 1.

\begin{tabular}{|c|c|c|}
\hline $\mathrm{n}$ & Error of RLG method & Error of LG method \\
\hline 3 & $285195 \times 10^{-4}$ & $120452 \times 10^{-4}$ \\
\hline 4 & $638431 \times 10^{-8}$ & $762999 \times 10^{-6}$ \\
\hline 5 & $346010 \times 10^{-13}$ & $527053 \times 10^{-10}$ \\
\hline 6 & $592489 \times 10^{-18}$ & $386018 \times 10^{-15}$ \\
\hline 7 & $961053 \times 10^{-21}$ & $294958 \times 10^{-18}$ \\
\hline
\end{tabular}

Experiment 2.Consider the following integral

$$
\int_{-0.5}^{0} \frac{x^{2}+1}{x^{3}+x+1} d x
$$

then the obtained results from RLG and LG methods are shown in table 2.

Table 2: Comparison between RLG and LG methods for computation of (25) of experiment 2.

\begin{tabular}{|c|c|c|}
\hline $\mathrm{n}$ & Error of RLG method & Error of LG method \\
\hline 3 & $411535 \times 10^{-4}$ & $102165 \times 10^{-4}$ \\
\hline 4 & $248111 \times 10^{-8}$ & $376287 \times 10^{-6}$ \\
\hline 5 & $214508 \times 10^{-13}$ & $9055275 \times 10^{-10}$ \\
\hline 6 & $375112 \times 10^{-18}$ & $253243 \times 10^{-15}$ \\
\hline 7 & $365112 \times 10^{-21}$ & $265643 \times 10^{-18}$ \\
\hline
\end{tabular}

\section{The operational matrices with respect to rational Laguerrefunctions (generalized)}

In this section, we obtain the operational matrices with respect to rational Laguerre functions. To do this, first we introduce the concept of operational matrix.

\subsection{The operational matrix}

Definition 1.Suppose

$$
\phi=\left[\phi_{0}, \phi_{1}, \ldots, \phi_{n}\right],(26)
$$

where $\phi_{0}, \phi_{1}, \ldots, \phi_{n}$ are the basis functions on the given interval $[a, b]$. The matrices $E_{n \times n}$ and $F_{n \times n}$ are named as the operational matrices of derivatives and integrals respectively if and only if

$$
\begin{aligned}
\frac{\mathrm{d}}{\mathrm{dt}} \phi(t) & \simeq E \phi(t), \\
\int_{a}^{x} \phi(t) d t & \simeq F \phi(t) .
\end{aligned}
$$

Further assume $\mathrm{g}=\left[\mathrm{g}_{0}, \mathrm{~g}_{1}, \ldots, \mathrm{g}_{n}\right]$, named as the operational matrix of the product, if and only if 


$$
\phi(x) \phi^{T}(x) \simeq G_{g} \phi(x) .
$$

In other words, to obtain the operational matrix of a product, it is sufficient to find $\mathrm{g}_{i, j, k}$, in the following relation

$$
\phi_{i}(x) \phi_{j}(x) \simeq \sum_{k=0}^{i+j} g_{i, j, k} \phi_{k}(x)
$$

which is called the linearization formula [19]. Operational matrices are used in several areasof numerical analysis and they hold particular importance in various subjects such as integral equations [20], differential and partial differential equations [21] and etc. Also many textbooks and papers have employed the operational matrices for spectral methods [9]. Now we present the following theorem.

Theorem 2.If we present $f(x)$ in the form

$$
f(x)=\sum_{k=0}^{\infty} D_{k}^{(\alpha)} R_{k}^{\alpha}(x, h), x \in[a, b),
$$

where

$$
D_{k}^{(\alpha)}=\left(\gamma_{k}^{\alpha}\right)^{-1}\left\langle f, R_{k}^{\alpha}(x, h)\right\rangle_{w_{r}^{\alpha}(x)} .
$$

Then

$$
\sum_{i=k}^{\infty} D_{i}^{(\alpha)} E_{k}^{(\alpha, i)}=\mu_{k}
$$

where

$$
\mu_{k}=\frac{g^{(k)}(0)}{k !}, g(x)=f\left(\frac{h(x-a)}{b-x}\right)
$$

and $E_{k}^{(\alpha, i)}$ is presented in (4).

Proof: If we use the change of variable $x \rightarrow \frac{h(x-a)}{b-x}$ in (3), then we have

$$
R_{n}^{(\alpha)}(x, h)=\sum_{k=0}^{n} E_{k}^{(\alpha, n)}\left(\frac{h(x-a)}{b-x}\right)^{k} .
$$

But (34), can be rewritten as

$$
\begin{aligned}
& f(x)=\sum_{k=0}^{\infty} D_{k}^{(\alpha)}\left(\sum_{i=0}^{k} E_{i}^{(\alpha, k)}\left(\frac{h(x-a)}{b-x}\right)^{i}\right)=\left(\sum_{i=0}^{\infty} D_{i}^{(\alpha)} E_{0}^{(\alpha, i)}\right)+\left(\sum_{i=1}^{\infty} D_{i}^{(\alpha)} E_{1}^{(\alpha, i)}\right)\left(\frac{h(x-a)}{b-x}\right) \\
& +\left(\sum_{i=2}^{\infty} D_{i}^{(\alpha)} E_{2}^{(\alpha, i)}\right)\left(\frac{h(x-a)}{b-x}\right)^{2}+\ldots=\sum_{k=0}^{\infty}\left(\sum_{i=k}^{\infty} D_{i}^{(\alpha)} E_{k}^{(\alpha, i)}\right)\left(\frac{h(x-a)}{b-x}\right)^{k} .
\end{aligned}
$$

Now if we define

$$
g(x)=f\left(\frac{b x+h a}{h+x}\right) \Rightarrow f(x)=g\left(\frac{h(x-a)}{b-x}\right),
$$

then from the Maclaurin series of $g(x)$, we have:

$$
g(x)=\sum_{k=0}^{\infty} \frac{g^{(k)}(0)}{k !} x^{k},
$$


or

$$
f(x)=g\left(\frac{h(x-a)}{b-x}\right)=\sum_{k=0}^{\infty} \frac{g^{(k)}(0)}{k !}\left(\frac{h(x-a)}{b-x}\right)^{k} .
$$

Comparing (35) and (38), we get

$$
\sum_{i=k}^{\infty} D_{i}^{(\alpha)} E_{k}^{(\alpha, \beta, i)}=\frac{g^{(k)}(0)}{k !}
$$

so the proof is completed.

Theorem 3.Suppose $R^{\alpha}(x, h)=\left[R_{0}^{\alpha}(x, h), R_{1}^{\alpha}(x, h), \ldots, R_{n}^{\alpha}(x, h)\right]$, where the elements $R_{i}^{\alpha}(x, h)$ are the rational Laguerre functions, then we have

$$
\frac{d}{d x}\left[R_{0}^{\alpha}(x, h), R_{1}^{\alpha}(x, h), \ldots, R_{n}^{\alpha}(x, h)\right] \simeq D_{(n+1) \times(n+1)}^{\alpha}\left[\begin{array}{c}
R_{0}^{\alpha}(x, h) \\
R_{1}^{\alpha}(x, h) \\
\vdots \\
R_{n}^{\alpha}(x, h)
\end{array}\right],
$$

where $D_{i}^{\alpha}$ (the ith-column of $D_{n \times n}^{\alpha}$ ) is obtained from this linear system (with upper triangular matrix)

$$
\left[\begin{array}{ccccc}
E_{0}^{(\alpha, 0)} & \ldots & E_{0}^{(\alpha, i-1)} & E_{0}^{(\alpha, i)} & E_{0}^{(\alpha, i+1)} \\
0 & E_{1}^{(\alpha, 1)} & \ldots & E_{1}^{(\alpha, i)} & E_{1}^{(\alpha, i+1)} \\
\vdots & \vdots & \ddots & \vdots & \vdots \\
0 & \ldots & E_{i-1}^{(\alpha, i-1)} & E_{i-1}^{(\alpha, i)} & E_{i-1}^{(\alpha, i+1)} \\
0 & 0 & 0 & E_{i}^{(\alpha, i)} & E_{i}^{(\alpha, i+1)} \\
0 & 0 & 0 & \ldots & E_{i+1}^{(\alpha, i+1)}
\end{array}\right]\left[\begin{array}{c}
d_{i, 0} \\
d_{i, 1} \\
\vdots \\
\vdots \\
d_{i, i} \\
d_{i, i+1}
\end{array}\right]=\left[\begin{array}{c}
\mu_{0} \\
\mu_{1} \\
\vdots \\
\vdots \\
\mu_{i} \\
\mu_{i+1}
\end{array}\right] .
$$

Proof:We know that there exist $\mathrm{d}_{i, j}$ such that:

$$
\frac{(h+x)^{2}\left(L_{i}^{\alpha}(x)\right)^{\prime}}{h b-h a}=\sum_{j=0}^{i+1} d_{i, j} L_{j}^{\alpha}(x),
$$

but if in (42), we apply the change of variable $x \rightarrow \frac{h(x-a)}{b-x}$, we have

$$
\frac{h b-h a}{(b-x)^{2}}\left(L_{i}^{\alpha}\left(\frac{h(x-a)}{b-x}\right)\right)^{\prime}=\sum_{j=0}^{i+1} d_{i, j}\left(L_{i}^{\alpha}\left(\frac{h(x-a)}{b-x}\right)\right)^{\prime},
$$

or

$$
\left(R_{i}^{\alpha}(x, h)\right)^{\prime}=\sum_{j=0}^{i+1} d_{i, j} R_{j}^{\alpha}(x, h)
$$

Combining (44) and (3), yields:

$$
\left(R_{i}^{\alpha}(x, h)\right)^{\prime}=\sum_{j=0}^{i+1} \sum_{k=0}^{j} d_{i, j} E_{k}^{(\alpha, j)}\left(\frac{h(x-a)}{b-x}\right)^{k} .
$$

Now noting to theorem 2 , in this case $\left(f(x)=R^{\prime}(x, h)\right)$, we get: 


$$
\sum_{j=k}^{i+1} d_{i, j} E_{k}^{(\alpha, j)}=\frac{g^{(k)}(0)}{k !}, k=0,1, \ldots, i+1,
$$

thus from (46), we can obtain the following linear system:

$$
\left[\begin{array}{ccccc}
E_{0}^{(\alpha, 0)} & \ldots & E_{0}^{(\alpha, i-1)} & E_{0}^{(\alpha, i)} & E_{0}^{(\alpha, i+1)} \\
0 & E_{1}^{(\alpha, 1)} & \ldots & E_{1}^{(\alpha, i)} & E_{1}^{(\alpha, i+1)} \\
\vdots & \vdots & \ddots & \vdots & \vdots \\
0 & \ldots & E_{i-1}^{(\alpha, i-1)} & E_{i-1}^{(\alpha, i)} & E_{i-1}^{(\alpha, i+1)} \\
0 & 0 & 0 & E_{i}^{(\alpha, i)} & E_{i}^{(\alpha, i+1)} \\
0 & 0 & 0 & \ldots & E_{i+1}^{(\alpha, i+1)}
\end{array}\right]\left[\begin{array}{c}
d_{i, 0} \\
d_{i, 1} \\
\vdots \\
\vdots \\
d_{i, i} \\
d_{i, i+1}
\end{array}\right]=\left[\begin{array}{c}
\mu_{0} \\
\mu_{1} \\
\vdots \\
\vdots \\
\mu_{i} \\
\mu_{i+1}
\end{array}\right] .
$$

Solving the linear system (47), concludes:

$$
\begin{gathered}
d_{i, i+1}=\frac{\mu_{i+1}}{E_{i+1}^{(\alpha, i+1)}}, \\
d_{i, j}=\frac{\mu_{i}-\sum_{k=i+1}^{j+1} E_{j}^{(\alpha, k)} d_{i, k}}{E_{j}^{(\alpha, j)}} ; j=0,1, \ldots, i .
\end{gathered}
$$

Also with similar strategy, we can obtain the integral operational matrix of rational Laguarrefunctions. This means if we consider

$$
\int_{0}^{u} \frac{(h+t)^{2}}{h b-h a} L_{i}^{\alpha}(t) d t=\sum_{j=0}^{i+3} c_{i, j} R_{j}^{\alpha}(u, h) ;,-1 \leq u \leq 1 .
$$

Employing the change of variable $t \rightarrow \frac{h(t-a)}{b-t}$, on right hand side of (49), gives:

$$
\int_{a}^{\frac{h(t-a)}{b-t}} L_{i}^{\alpha}\left(\frac{h(t-a)}{b-t}\right) d t=\sum_{j=0}^{i+3} c_{i, j} L_{j}^{\alpha}(u) .
$$

Now if we define $x=\frac{b t-h a}{t+h}$ then (50) transforms to

$$
\int_{a}^{x} L_{i}^{\alpha}\left(\frac{t-1}{t+1}\right) d t=\sum_{j=0}^{i+3} c_{i, j} L_{j}^{\alpha}\left(\frac{x-1}{x+1}\right)
$$

or

$$
\int_{a}^{x} R_{i}(t, h) d t=\sum_{j=0}^{i+3} c_{i, j} R_{j}(x, h) .
$$

Combining (52) and (3), yields

$$
\int_{0}^{x} R_{i}^{\alpha}(t, h) d t=\sum_{j=0}^{i+3} \sum_{k=0}^{j} c_{i, j} E_{k}^{(\alpha, j)}\left(\frac{h(x-a)}{b-x}\right)^{k} .
$$

Now noting to theorem 2, we get:

$$
\sum_{j=k}^{i+3} c_{i, j} E_{k}^{(\alpha, \beta, j)}\left(\frac{h(x-a)}{b-x}\right)^{k}=\mu_{k} ; k=0,1, \ldots, i+3 .
$$

From (54), we obtain the following linear system: 


$$
\left[\begin{array}{ccccc}
E_{0}^{(\alpha, 0)} & \ldots & E_{0}^{(\alpha, i+1)} & E_{0}^{(\alpha, i+2)} & E_{0}^{(\alpha, i+3)} \\
0 & E_{1}^{(\alpha, 1)} & \ldots & E_{1}^{(\alpha, i+2)} & E_{1}^{(\alpha, i+3)} \\
\vdots & \vdots & \ddots & \vdots & \vdots \\
0 & \ldots & E_{i-1}^{(\alpha, i+1)} & E_{i-1}^{(\alpha, i+2)} & E_{i-1}^{(\alpha, i+3)} \\
0 & 0 & 0 & E_{i+2}^{(\alpha, i+2)} & E_{i+2}^{(\alpha, i+3)} \\
0 & 0 & 0 & \ldots & E_{i+3}^{(\alpha, i+3)}
\end{array}\right]\left[\begin{array}{c}
c_{i, 0} \\
c_{i, 1} \\
\vdots \\
\vdots \\
c_{i, i+2} \\
c_{i, i+3}
\end{array}\right]=\left[\begin{array}{c}
\mu_{0} \\
\mu_{1} \\
\vdots \\
\vdots \\
\mu_{i+2} \\
\mu_{i+3}
\end{array}\right] .
$$

Solving the linear system (55), concludes:

$$
\begin{gathered}
c_{i, i+3}=\frac{\mu_{3+1}}{E_{i+3}^{(\alpha, i+3)}}, \\
c_{i, j}=\frac{\mu_{i}-\sum_{k=i+1}^{j+1} E_{j}^{(\alpha, k)} c_{i, k}}{E_{j}^{(\alpha, j)}} ; j=0,1, \ldots, i+2 .
\end{gathered}
$$

Therefore

$$
\int\left[R_{1}^{\alpha}(x, h), R_{2}^{\alpha}(x, h), \ldots, R_{n}^{\alpha}(x, h)\right] d x \simeq I_{(n+1) \times(n+1)}^{\alpha}\left[\begin{array}{c}
R_{1}^{\alpha}(x, h) \\
R_{1}^{\alpha}(x, h) \\
\vdots \\
R_{1}^{\alpha}(x, h)
\end{array}\right],
$$

in which (the ith-column of $I_{n \times n}^{\alpha}$ is obtained from the linear system (55)). Finally for obtaining the operational matrix of product, we use these two important formulas

$$
L_{n}^{\alpha}(x) L_{m}^{\beta}(x)=\sum_{i=0}^{m+n} c_{i}(m, n, \alpha, \beta) L_{m}^{\alpha+\beta}(x),
$$

and

$$
L_{n}^{\alpha}(x)=\sum_{i=0}^{n}\left(\begin{array}{c}
\alpha-\beta+n-i+1 \\
n-i
\end{array}\right) L_{i}^{\beta}(x),
$$

where

$$
c_{i}(m, n, \alpha, \beta)=(-1)^{m+n+\alpha} \sum_{k=0}^{i}\left(\begin{array}{l}
i \\
k
\end{array}\right)\left(\begin{array}{c}
m+\alpha \\
n-k+i
\end{array}\right)\left(\begin{array}{l}
n+\alpha \\
m-k
\end{array}\right) .
$$

By combining the relations (58) and (59), we obtain

$$
L_{n}^{\alpha}(x) L_{m}^{\alpha}(x)=\sum_{i=0}^{m+n} M_{i}(m, n, \alpha) L_{k}^{\alpha}(x)
$$

where

$$
M_{i}(m, n, \alpha)=\sum_{k=0}^{i} d_{i}(m, n, \alpha)\left(\begin{array}{c}
\alpha+i-k+1 \\
i-k
\end{array}\right) \text {, }
$$

and

$$
d_{i}(m, n, \alpha)=c_{i}(m, n, \alpha, \alpha) \sum_{k=0}^{i}\left(\begin{array}{c}
\alpha+i-k+1 \\
i-k
\end{array}\right) \text {. }
$$


Using the change of variable $x \rightarrow \frac{h(x-a)}{b-x}$ we obtain

$$
R_{n}^{\alpha}(x, h) R_{m}^{\alpha}(x, h)=\sum_{i=0}^{m+n} d_{i}(m, n, \alpha) \sum_{k=0}^{i}\left(\begin{array}{c}
\alpha+i-k+1 \\
i-k
\end{array}\right) R_{k}^{\alpha}(x, h) .
$$

Therefore

$$
\left[R_{0}^{\alpha}(x, h), R_{1}^{\alpha}(x, h), \ldots, R_{n}^{\alpha}(x, h)\right]\left[\begin{array}{c}
R_{0}^{\alpha}(x, h) \\
R_{1}^{\alpha}(x, h) \\
\vdots \\
R_{n}^{\alpha}(x, h)
\end{array}\right] \simeq P_{(n+1) \times(1)}^{\alpha}\left[\begin{array}{c}
R_{0}^{\alpha}(x, h) \\
R_{1}^{\alpha}(x, h) \\
\vdots \\
R_{n}^{\alpha}(x, h)
\end{array}\right],
$$

where $P_{i, 1}^{\alpha}=\sum_{j=0}^{n-i} M(j, j, \alpha)$

\section{Approximation with rational Laguerre functions (generalized)}

Now we present some approximation properties of rational Laguerre functions. Let us considerthe finite dimensional approximation space

$$
R_{n}^{h}=\operatorname{span}\left\{R_{i}^{\alpha}(x, h), i=0, \ldots, n\right\},
$$

in which $R_{i}^{\alpha}(x, h)$ are orthogonal with respect to $w_{r}^{\alpha}$ (defined on $\left.[a, b)\right)$ and consider the orthogonal projection $P_{n}^{(R, h, \alpha)}(u): L_{w_{r}^{\alpha}[a, b)}^{2} \rightarrow \chi_{n}$ such that

$$
\left(P_{n}^{(R, h, \alpha)}(u)-u, v_{n}\right)_{w}=0, \forall v_{n} \in R_{n}^{h}
$$

From (67) and the orthogonality relation of rational Laguerre functions, we can write

$$
P_{n}^{(R, h, \alpha)}(u)=\sum_{n=0}^{N} u_{n} R_{n}^{\alpha}(x, h)
$$

where

$$
u_{n}=\frac{1}{\gamma_{n}} \int_{a}^{b} u(x) R_{n}^{\alpha}(x, h) w(x) d x .
$$

Now consider the general operator equation

$$
\mathcal{L} u+N u=g(x), x \in[a, b),
$$

with respect to boundary condition

$$
\mathcal{B} u=\lambda
$$

where $\mathcal{L}, \mathcal{N}$ and $\mathcal{B}$ are the linear, nonlinear and boundary operators respectively related to a suitable Hilbert space, $[a, b)$ is the domain (one dimensional) of approximation, $\lambda$ is an arbitrary constant and 
$g(x) \in L_{w_{r}^{\alpha}}^{2}([a, b))$ is an arbitrary function. Let $R_{n}^{h}$ be an approximation space with weight function $w_{r}^{\alpha}(x)$ and a set of collocation points $\left\{x_{j}\right\}_{j=0}^{n}$, shifted roots of Laguerre polynomial with degree $n$. he spectralcollocation method for the operator equation (70) defined in the following form: Find $\phi(x) \in R_{n}^{h}$ or $\phi(x)=\sum_{i=0}^{n} a_{i} R_{i}^{\alpha}(x, h)$ such that

$$
\begin{gathered}
\mathcal{L} \phi\left(x_{j}\right)+N \phi\left(x_{j}\right)=g\left(x_{j}\right), j=0, \ldots, n, \\
\mathcal{B}(\phi(x))=\lambda .
\end{gathered}
$$

If we suppose that the boundary conditions give us the $m$ equations, then from equation (72), we obtain $m+n+1$ algebraic equations and so for obtaining the $n+1$ unknowns, we must eliminate the $m$ equation and add boundary conditions, which must be solved for unknown $\left\{a_{i}\right\}_{i=0}^{n}$ coefficients. Also the spectralGalerkin method for the operator equation (70) is defined in the following form:

Find $\phi(x) \in R_{n}^{h}$ or $\phi(x)=\sum_{i=0}^{n} a_{i} R_{i}^{\alpha}(x, h)$ such that $\phi(x)$ are satisfied in the boundary conditions (71) automatically and

$$
(\mathcal{L} \phi(x), p(x))_{w_{r}^{\alpha}}+(N \phi(x), p(x))_{w_{r}^{\alpha}}=(g(x), p(x))_{w_{r}^{\alpha}}, \forall p(x) \in R_{n}^{h} .
$$

Also from equation (73), we obtain $n+1$ algebraic equations which must be solved for unknown $\left\{a_{i}\right\}_{i=0}^{n}$ coefficients. In this work, by using MATLAB software (version 2013), we solve these algebraic systems.

\subsection{The error analysis}

Now in this section, we present the details of the convergence rates of rational Laguerre functions (generalized) as basic functions. First we consider the following theorem.

Theorem 4.For any $f \in B_{m}^{\alpha}(0,+\infty)$ and $m \geq 0$,

$$
\left\|\frac{d^{l}}{d x}\left(P_{N}^{(\alpha)}(f)-f\right)\right\|_{w_{\alpha+l}} \leq c N^{\left(\frac{l-m}{2}\right)}\left\|\frac{d^{m}}{d x^{m}}(u)\right\|_{w_{\alpha+m}}, 0 \leq l \leq m,
$$

where

$$
B_{m}^{\alpha}(0, \infty)=\left\{f: \frac{d^{k}}{d x^{k}}(f) \in L_{w_{\alpha+k}}^{2}(0, \infty), 0 \leq k \leq m\right\},
$$

$P_{n}^{(\alpha)}$ is the $L_{w^{\alpha}(x)}^{2}$-orthogonal projection operator associated with the Laguerre polynomials(generalized) and $c$ is a positive constant.

Proof: See [13]. 
Theorem 5. For any $u \in G_{\alpha}^{m}([a, b))$ with $m \geq 0$

$$
\left\|P_{n}^{(R, h, \alpha)}(u)-u\right\|_{w_{r}^{\alpha}(x)} \leq c N^{-m / 2}\left\|\frac{D^{m}}{D x^{m}}(u)\right\|_{w_{r}^{\alpha+m}},
$$

and for $m \geq 1$,

$$
\left\|\frac{d}{d x}\left(P_{N}^{(R, h, \alpha)}(u)-u\right)\right\|_{w_{r}^{\alpha+1}(x)} \leq c N^{(1-m) / 2}\left\|\frac{D^{m}}{D x^{m}}(u)\right\|_{w_{r}^{\alpha+m}},
$$

where

$$
G_{\alpha}^{m}([a, b))=\left\{u: \frac{d^{k}}{d x^{k}}(u) \in L_{w_{r}^{\alpha+k}}^{2}([a, b)), 0 \leq k \leq m\right\},
$$

$c$ is a positive constant and the operator $\frac{D^{k}}{D x^{k}}(u)$ is defined as

$$
\frac{D^{k}}{D x^{k}}(u)=\underbrace{\left(\frac{h(x-a)}{b-x}\right)^{\prime} \frac{d}{d x}\left(\left(\frac{h(x-a)}{b-x}\right)^{\prime} \frac{d}{d x}\left(\ldots .\left(\left(\frac{h(x-a)}{b-x}\right)^{\prime} \frac{d}{d x}\right) \ldots\right)\right)}_{\text {t times }} .
$$

Proof: Let $U_{h}(x)=\sum_{i=0}^{\infty} U_{h, i} L_{i}^{\alpha}(x)$ and $u_{h}(x)=\sum_{i=0}^{\infty} u_{h, i} R_{i}^{\alpha}(x, h)$, then from definition, it is easyto show the relation between the coefficients of the rational Laguerre functions (generalized) and Laguerre polynomials expansions (generalized) as

$$
u_{h, i}=\frac{\left\langle u, R_{i}^{\alpha}(x, h)\right\rangle_{w_{r}^{\alpha}(x)}}{\gamma_{i}^{\alpha}}=\frac{\left\langle u, L_{i}^{\alpha}(x)\right\rangle_{w_{\alpha}(x)}}{\gamma_{i}^{\alpha}}=U_{h, i} .
$$

Also we need for expressing the error estimates, to introduce an operator

Repeating formula (79) leads to

$$
\frac{D}{D x}(u)=\left(\frac{h(x-a)}{b-x}\right)^{\prime} \frac{d u}{d x} \text {. }
$$

$$
\frac{d^{k} U_{h}(x)}{d x^{k}}=\underbrace{\left(\frac{h(x-a)}{b-x}\right)^{\prime} \frac{d}{d x}\left(\left(\frac{h(x-a)}{b-x}\right)^{\prime} \frac{d}{d x}\left(\ldots .\left(\left(\frac{h(x-a)}{b-x}\right)^{\prime} \frac{d}{d x}\right) \ldots .\right)\right.}_{\text {ktimes }}=\frac{D^{k}}{D x^{k}}(u) .
$$

Let $P_{n}^{(\alpha)}$ be the $L_{w^{\alpha}(x)}^{2}$ - orthogonal projection operator associated with the Laguerre polynomials (generalized), then by Parseval identity [7], theorem (3) and formula (82), we have 


$$
\begin{aligned}
& \left\|P_{N}^{(R, h, \alpha)}(u)-u\right\|_{w_{r}^{\alpha}(x)}^{2}=\sum_{i=N+1}^{\infty}\left(u_{h, i}\right)^{2} \gamma_{i}^{\alpha}=\sum_{i=N+1}^{\infty}\left(U_{h, i}\right)^{2} \gamma_{i}^{\alpha} \\
& =\left\|P_{N}^{(\alpha)}\left(U_{s}\right)-U_{s}\right\|_{w^{\alpha}(x)}^{2} \leq c N^{-m}\left\|U_{s}^{(m)}\right\|_{w^{\alpha}(x)}^{2} \leq c N^{-m}\left\|\frac{D^{k}}{D x^{k}}(u)\right\|_{w_{r}^{\alpha}(x)}^{2} .
\end{aligned}
$$

Next, we deduce from (7) (orthogonality of $\left\{\left(L_{i}^{\alpha}(x)\right)^{\prime}\right\}$ that $\left\{\left(R_{i}^{\alpha}(x, h)\right)^{\prime}\right\}$ is $L_{w_{r}^{\alpha}}^{2}$ orthogonal, and

$$
\left\|\left(R_{i}^{\alpha}(x, h)\right)^{\prime}\right\|_{w_{r}^{\alpha}}^{2}=\left\|\left(L_{i}^{\alpha}(x)\right)^{\prime}\right\|_{w^{\alpha}}^{2}=\lambda_{i}^{\alpha} \gamma_{i}^{\alpha},
$$

where $\lambda_{i}^{\alpha}$ is the eigenvalue of the Laguerre Sturm-Liouville problem (1). Therefore by (80) and theorem (3), we have

$$
\begin{array}{r}
\left\|\frac{d}{d x}\left(P_{N}^{(R, h, \alpha)}(u)-u\right)\right\|_{w_{r}^{\alpha}}^{2}=\sum_{i=N+1}^{\infty} \lambda_{i}^{\alpha} \gamma_{i}^{\alpha}\left(u_{h, i}\right)^{2}=\sum_{i=N+1}^{\infty} \lambda_{i}^{\alpha} \gamma_{i}^{\alpha}\left(U_{h, i}\right)^{2}=\left\|\frac{d}{d x}\left(P_{N}^{(\alpha)}(u)-u\right)\right\|_{w^{\alpha}}^{2} \leq \\
c N^{(1-m)}\left\|u^{(m)}\right\|_{w^{\alpha+1}}^{2} \leq c N^{(1-m)}\left\|\frac{D^{m}}{D x^{m}} u\right\|_{w^{\alpha+m}}^{2} .
\end{array}
$$

Thus the proof is completed.

Now we present a theorem which express the error analysis of projection operator of exact solution of (70) based on Galerkin basis functions.

Theorem 6. Suppose $\mathcal{L}$ and $\mathcal{N}$ are the linear and nonlinear operators with respect to (70) wherein $\mathcal{L}$ has inversion, $\mathcal{L}^{-1}$ and $\mathcal{N}$ have these properties

$$
\begin{gathered}
\|\mathcal{N}(x)-\mathcal{N}(y)\| \leq\|\mathcal{N}|\|\mid x-y\|, \\
\left\|\mathcal{L}^{-1}(x)\right\| \leq\left\|\mathcal{L}^{-1}|\|\mid x\|,\right.
\end{gathered}
$$

and $f, g \in G_{\alpha}^{m}([a, b)),(m \geq 1)$ are satisfied in equation (70), then

$$
\left\|f-P_{N}^{(R, h, \alpha)}(f)\right\|_{w_{r}^{(\alpha)}} \leq c\left(1-\left\|L^{-1}\right\|\|N\|\right) T,
$$

where

$$
T=N^{-m / 2}\left\|\mathcal { L } ^ { - 1 } \left|\left\|\mid \frac{D^{m}}{D x^{m}}\left(N\left(P_{N}^{(R, h, \alpha)}(f)\right)\right)\right\|_{w_{r}^{\alpha+m}} .\right.\right.
$$

Proof: From equation (70) and inevitability of $\mathcal{L}$ we have

$$
f+\mathcal{L}^{-1} \mathcal{N} f=\mathcal{L}^{-1}(g)+k(x),(90)
$$

where $k(x)$ is a function dependent on an operator $\mathcal{L}$. Now by substituting $P_{N}^{(R, h, \alpha)}(f)$ in (90) we have

$$
P_{N}^{(R, h, \alpha)}(f)+\mathcal{L}^{-1} \mathcal{N} P_{N}^{(R, h, \alpha)}(f) \simeq \mathcal{L}^{-1}(g)+k(x) .
$$

Also the nonlinear term $\mathcal{N}\left(P_{N}^{(R, h, \alpha)}(f)\right)$ is approximated by

$$
\mathcal{N} P_{N}^{(R, h, \alpha)}(f) \simeq P_{N}^{(R, h, \alpha)}\left(\mathcal{N} P_{N}^{(R, h, \alpha)}(f)\right),
$$

so from (92) and (91), we obtain

$$
P_{N}^{(R, h, \alpha)}(f)+\mathcal{L}^{-1} P_{N}^{(R, h, \alpha)}\left(\mathcal{N} P_{N}^{(R, h, \alpha)}(f)\right) \simeq \mathcal{L}^{-1}(g)+k(x) .
$$

Now by subtracting (90) and (93), we have

$$
\left(f-P_{N}^{(R, h, \alpha)}(f)\right) \simeq\left(\mathcal{L}^{-1} P_{N}^{(R, h, \alpha)}\left(N P_{N}^{(R, h, \alpha)}(f)\right)-\mathcal{L}^{-1} \mathcal{N}(f)\right) .
$$


From (94), we have

$$
\begin{aligned}
\left(f-P_{N}^{(R, h, \alpha)}(f)\right) \simeq & \mathcal{L}^{-1} P_{N}^{(R, h, \alpha)}\left(\mathcal{N} P_{N}^{(R, h, \alpha)}(f)\right)- \\
& \mathcal{L}^{-1} \mathcal{N} P_{N}^{(R, h, \alpha)}(f)+\mathcal{L}^{-1} \mathcal{N} P_{N}^{(R, h, \alpha)}(f)-\mathcal{L}^{-1} \mathcal{N}(f),
\end{aligned}
$$

so using properties (86) and (87), we obtain

$$
\begin{aligned}
& \left\|f-P_{N}^{(R, h, \alpha)}(f)\right\|_{w_{r}^{\alpha}} \simeq\left\|\mathcal{L}^{-1}\left(P_{N}^{(R, h, \alpha)}\left(\mathcal{N} P_{N}^{(R, h, \alpha)}(f)\right)-\mathcal{N} P_{N}^{(R, h, \alpha)}(f)\right)\right\| \\
& +\left\|\mathcal{L}^{-1}\left(\mathcal{N} P_{N}^{(R, h, \alpha)}(f)-\mathcal{N}(f)\right)\right\| \leq\left\|\mathcal{L}^{-1}\right\|\left\|\left(P_{N}^{(R, h, \alpha)}\left(\mathcal{N} P_{N}^{(R, h, \alpha)}(f)\right)-\mathcal{N} P_{N}^{(R, h, \alpha)}(f)\right)\right\|+ \\
& \left\|\mathcal{L}^{-1}\right\|\|\mathcal{N}\|\left\|P_{N}^{(R, h, \alpha)}(f)-(f)\right\| .
\end{aligned}
$$

Finally using theorem (4), we obtain

$$
\left\|f-P_{N}^{(R, h, \alpha)}(f)\right\|_{w_{r}(\alpha)} \leq c\left(1-\left\|\mathcal{L}^{-1}\right\|\|\mathcal{N}\|\right) T,
$$

where

$$
T=N^{-m / 2}\left\|\mathcal { L } ^ { - 1 } \left|\left\|\mid \frac{D^{m}}{D x^{m}} N\left(P_{N}^{(R, h, \alpha)}(f)\right)\right\|_{w_{r}^{\alpha+m}} .\right.\right.
$$

Thus the proof is completed.

Remark 2.In the similar discussion, we can obtain a new rational basis functions in the interval $(a, b)$ using Hermit polynomials [5] and transform function

$$
y=\frac{h x}{\sqrt{(x-a)(b-x)}},
$$

in which is named as an algebraic transformation, and transform the interval $y \in(-\infty,+\infty)$ to $x \in[a, b)$.

\section{The test experiment}

Functions with stiffness structures arise in many applied sciences such as in engineering, physics and applied mathematics. For instance, when exact solution of problems contains terms of the form $e^{k x}$, where $\mathrm{k}$ is a negative real part of a complex number problem, gives meaningless results [22-28]. In general, the methods designed for non-stiff problems such as spectral methods (Galerkin, Tau and collocation and based on classical polynomials) [7-11], homotopy analysis method [29], vibrational iteration method [30-32] when applied to stiff systems of differential equations tend to be very slow and can give bad (divergent) results in solution. Now in this section, a test experiment is presented where its solution has stiffness structure. In this case, using spectral collocation method based on classical Jacobi polynomials with free parameters $(\alpha, \beta)$ we obtain very bad results in global interval $[0,1]$, because of the stiffness structures of the exact solutions although if we use adaptive spectral collocation method based on classical Jacobi polynomials in sub-intervals with small distances, we can obtain better results. But using spectral method based on new rational basis functions, we show the accuracy and superiority of our new basis functions rather than other basis functions such as polynomials in global interval.

Experiment 3.Consider the following system of differential equations [33], our new basis functions rather than other basis functions such as polynomials in global interval. 


$$
\left[\begin{array}{l}
x^{\prime}(t) \\
y^{\prime}(t) \\
z^{\prime}(t)
\end{array}\right]=\left(\begin{array}{ccc}
-21 & 19 & -20 \\
19 & -21 & 20 \\
40 & -40 & 40
\end{array}\right)\left[\begin{array}{l}
x(t) \\
y(t) \\
z(t)
\end{array}\right]
$$

with initial condition $x(0)=\left[\begin{array}{c}1.0 \\ 0.0 \\ -1\end{array}\right]$.

The exact solution is

$$
\begin{aligned}
& x(t)=\frac{1}{2}\left[e^{2 t}+e^{-40 t}(\cos (40 t)+\sin (40 t))\right], \\
& y(t)=\frac{1}{2}\left[e^{-2 t}-e^{-40 t}(\cos (40 t)-\sin (40 t))\right], \\
& z(t)=\frac{-1}{2}\left[e^{-2 t}+e^{-40 t}(\cos (40 t)-\sin (40 t))\right] .
\end{aligned}
$$

Now we approximate the exact solution of (100) based on rational Laguarre functions (generalized), or

$$
\begin{aligned}
& x(t) \simeq \sum_{i=0}^{m} \varepsilon_{i} R_{i}^{\alpha}(t, h)=\left(R^{\alpha, m}(x, h)\right)^{T} \varepsilon, \\
& y(t) \simeq \sum_{i=0}^{n} \beta_{i} R_{i}^{\alpha}(t, h)=\left(R^{\alpha, n}(x, h)\right)^{T} \beta, \\
& z(t) \simeq \sum_{i=0}^{p} \gamma_{i} R_{i}^{\alpha}(t, h)=\left(R^{\alpha, p}(x, h)\right)^{T} \gamma,
\end{aligned}
$$

where $\varepsilon_{i}, \beta_{i}, \gamma_{i}$, are the unknown coefficients,

$$
\varepsilon=\left[\varepsilon_{0}, \ldots, \varepsilon_{m}\right]^{T}, \beta=\left[\beta_{0}, \ldots, \beta_{n}\right]^{T}, \gamma=\left[\gamma_{0}, \ldots, \gamma_{p}\right]^{T},
$$

and

$$
R^{\alpha, i}(x, h)=\left[R_{0}^{\alpha}(x, h), R_{1}^{\alpha}(x, h), \ldots, R_{i}^{\alpha}(x, h)\right]^{T} .
$$

In this work, we assume $m=n=p$, and we can consider the matrix form of equation (100) as follows

$$
\begin{aligned}
& \left(R^{\alpha, n}(x, h)\right)^{T}\left(\left(D^{(\alpha)}+21 I_{n \times n}\right) \varepsilon-19 \beta+20 \gamma\right) \simeq 0, \\
& \left(R^{\alpha, n}(x, h)\right)^{T}\left(-19 \varepsilon-\left(D^{(\alpha)}+21 I_{n \times n}\right) \beta-20 \gamma\right) \simeq 0, \\
& \left(R^{\alpha, n}(x, h)\right)^{T}\left(-40 \varepsilon+40 \beta+\left(D^{(\alpha)}-40 I_{n \times n}\right) \gamma\right) \simeq 0,
\end{aligned}
$$

or

$$
\left.H^{(\alpha, n)}(x, h)\left(\begin{array}{ccc}
\left(D^{(\alpha)}+21 I_{n \times n}\right) & -19 I_{n \times n} & 20 I_{n \times n} \\
-19 I_{n \times n} & -\left(D^{(\alpha)}+21 I_{n \times n}\right) & -20 I_{n \times n} \\
-40 I_{n \times n} & 40 I_{n \times n} & \left(D^{(\alpha)}-40 I_{n \times n}\right.
\end{array}\right)\right)\left[\begin{array}{l}
\varepsilon \\
\beta \\
\gamma
\end{array}\right] \simeq\left[\begin{array}{l}
0 \\
0 \\
0
\end{array}\right],
$$


where

$$
H^{(\alpha, n)}(x, h)=\left[\left(R^{\alpha, n}(x, h)\right)^{T},\left(R^{\alpha, n}(x, h)\right)^{T},\left(R^{\alpha, n}(x, h)\right)^{T}\right],
$$

and $D^{(\alpha)}, I_{n \times n}$ are operational matrices of derivative and identity of order $\mathrm{n}$, respectively. Also the matrix form of initial conditions is in the following form

$$
H^{(\alpha, n)}(0, h)\left[\begin{array}{l}
\varepsilon \\
\beta \\
\gamma
\end{array}\right]=\left[\begin{array}{l}
1 \\
0 \\
-1
\end{array}\right] .
$$

Now we choose $n+1$ collocation points $x_{i}, i=0, \ldots, n$; and by vanishing (106) in these collocation points, we obtain $3 n$ algebraic equations for $3 n$ unknown coefficients. But for imposing the initial conditions, we eliminate the 3 latter equations of linear system (106) and add the 3 initial conditions (108). The obtained algebraic system is linear and solving it, is easy from implementation point of view.

Table 3: The comparison between our method and exact solutions of $x(t)$ for $\alpha=0, h=1, n=20$.

\begin{tabular}{|c|c|c|c|}
\hline $\mathrm{t}$ & Exact solution & Our method & Error \\
\hline 0.1 & 0.9959322154 & 0.9959322153 & 0.0000000001 \\
\hline 0.2 & 0.9876494697 & 0.9876494696 & 0.0000000001 \\
\hline 0.3 & 0.9757613240 & 0.9757613237 & 0.0000000003 \\
\hline 0.4 & 0.9608307308 & 0.9608307306 & 0.0000000002 \\
\hline 0.5 & 0.9433749893 & 0.9433749894 & 0.0000000001 \\
\hline 0.6 & 0.9238669934 & 0.9238669939 & 0.0000000005 \\
\hline 0.7 & 0.9027367174 & 0.9027367204 & 0.0000000030 \\
\hline 0.8 & 0.8803729025 & 0.8803729145 & 0.0000000120 \\
\hline 0.9 & 0.8571248954 & 0.8571249329 & 0.0000000375 \\
\hline 1.0 & 0.8333046046 & 0.8333047113 & 0.0000001067 \\
\hline
\end{tabular}

Table 4: The comparison between our method and exact solutions of $x(t)$ for $\alpha=0, h=4, n=20$.

\begin{tabular}{|c|c|c|c|}
\hline $\mathrm{t}$ & Exact solution & Our method & Error \\
\hline 0.1 & 0.9959322154 & 0.9959322171 & 0.0000000016 \\
\hline 0.2 & 0.9876494697 & 0.9876494711 & 0.0000000041 \\
\hline 0.3 & 0.9757613240 & 0.9757613251 & 0.0000000011 \\
\hline 0.4 & 0.9608307308 & 0.9608307311 & 0.0000000003 \\
\hline 0.5 & 0.9433749893 & 0.9433749904 & 0.0000000011 \\
\hline 0.6 & 0.9238669934 & 0.9238669937 & 0.0000000003 \\
\hline 0.7 & 0.9027367174 & 0.9027367195 & 0.0000000021 \\
\hline 0.8 & 0.8803729025 & 0.8803729136 & 0.0000000111 \\
\hline 0.9 & 0.8571248954 & 0.857124941 & 0.0000000456 \\
\hline 1.0 & 0.8333046046 & 0.833304619 & 0.0000000073 \\
\hline
\end{tabular}


Table 5: First 11 values of the coefficients of the rational Laguerre functions for $h=1, n=20$.

\begin{tabular}{|c|c|c|c|}
\hline $\mathrm{i}$ & $\varepsilon_{i}$ & $\beta_{i}$ & $\gamma_{i}$ \\
\hline 0 & -1.6654612769138686 & -3.0763080674131122 & -4.4121404005155346 \\
\hline 1 & -2.2218571378156790 & 6.2265759568134613 & -3.1437532589118954 \\
\hline 2 & 1.3180582498315322 & 8.3708382606137689 & 1.5931995757133679 \\
\hline 3 & 3.1675219015152311 & -7.6214127251131138 & -6.2144913908193678 \\
\hline 4 & $-6.8691591664 \times 10^{-2}$ & $2.5737469630 \times 10^{-2}$ & $-3.7064636669 \times 10^{-2}$ \\
\hline 5 & $4.9056578736 \times 10^{-3}$ & $-5.4056930803 \times 10^{-2}$ & $2.2524539084 \times 10^{-2}$ \\
\hline 6 & $3.5456430874 \times 10^{-3}$ & $6.2144913908 \times 10^{-3}$ & $-7.2889778446 \times 10^{-3}$ \\
\hline 7 & $-8.7714243647 \times 10^{-4}$ & $-7.5522515012 \times 10^{-3}$ & $-5.387825557 \times 10^{-3}$ \\
\hline 8 & $-2.7413862076 \times 10^{-4}$ & $-3.3948089920 \times 10^{-3}$ & $4.1803381700 \times 10^{-4}$ \\
\hline 9 & $2.7413862076 \times 10^{-4}$ & $2.2762628605 \times 10^{-4}$ & $2.1066422023 \times 10^{-4}$ \\
\hline 10 & $2.7413862076 \times 10^{-5}$ & $3.2741956094 \times 10^{-5}$ & $3.8591663085 \times 10^{-5}$ \\
\hline
\end{tabular}

\section{Conclusion}

In this work, we have presented a new rational basis functions defined on $[a, b)$ and based on mapping Laguerre polynomials to bounded domain $[a, b)$. Using these rational functions as basic functions, we have implemented spectral methods for numerical solution of operator equations and we have shown that in some cases (operator equations with fractional solutions), these basis functions are obtained better results than other basis functions (experiment 3). Also the quadrature formulae and operational matrices (derivative, integral and product) with respect to these basis functions are obtained. The importance and superiority of the new basis functions rather than other basis functions such as polynomials is shown for quadrature formulae and for numerical solution of operator equations in some experiments (experiments 1 and 2).

\section{References}

[1] W. Gautschi, "Orthogonal Polynomials (Computation and Approximation)", Oxford University Press, (2004).

[2] C.F. Dunkl, Y. Xu, “Orthogonal Polynomials of Several Variables”, Cambridge University Press, (2001).

[3] F. Marcellan, W. V. Assche, “Orthogonal Polynomials and Special Functions (a Computation and Applications)", Springer-Verlag Berlin Heidelberg, (2006).

[4] R. Askey, "Orthogonal Polynomials and Special Functions", SIAM-CBMS, Philadelphia, (1975).

[5] D. Gottlieb, S.A. Orszag, "Numerical Analysis of Spectral Methods: Theory and Applications", SIAM-CBMS, Philadelphia, (1977).

[6] J.P. Boyd, Chebyshev, "Fourier Spectral Methods", Dover Publications, Inc, New York, (2000).

[7] C. Canuto, M. Y. Hussaini, A. Quarteroni, T. A. Zang, "Spectral Methods: Fundamentals in Single Domains", Springer-Verlag, (2006).

[8] C. Canuto, M. Y. Hussaini, A. Quarteroni, T. A. Zang, "Spectral Method in Fluid Dynamics", Prentice Hall, Englewood Cliffs, NJ, (1984).

[9] L.N. Trefethen, "Spectral Methods in Matlab", SIAM, Philadelphia, PA, (2000).

[10] J.S. Hesthaven, S. Gottlieb, D. Gottlieb, "Spectral Methods for Time-Dependent Problems", Springer, (2009).

[11] G. Ben-yu, “The State of Art in Spectral Methods", Hong kong University, (1996). 
[12] J. Shen, T. Tang, Li-Lian Wang, "Spectral Methods Algorithms, Analysis and Applications", Springer, (2011).

[13] J. Shen, Li-Lian Wang, "Some Recent Advances on Spectral Methods for Un-bounded Domains", J. Commun. comput. Phys, 5 (2009) 195-241.

[14] D.A. Kopriva, "Implementing Spectral Methods for Partial Differential Equations", firsted, Cambridge University, (2007).

[15] A. Imani, A. Aminataei, A. Imani, "Collocation method via Jacobi polynomials for solving nonlinear ordinary differential equations”, Int. J. Math. Math. Sci., Article ID 673085 (2011), $11 \mathrm{P}$.

[16] K. Parand, M. Shahini, "Rational Chebyshev pseudo spectral approach for solving Thomas Fermi equation”, Physics Letters A, 373 (2009) 210-213.

[17] K. Parand, M. Shahini, M. Dehghan, "Rational Legendre pseudo spectral approach for solving nonlinear differential equations of Lane Emden type", J. Computational Physics, 228 (2009) 8830-8840.

[18] J.P. Boyd, "Rational Chebyshev spectral methods for unbounded solutions on an infinite interval using polynomial-growth special basis functions", Computers and Mathematics with Applications, 41 (2001) 1293-1315.

[19] M.R. Eslahchi, M. Dehghan, "Application of Taylor series in obtaining the orthogonal operational matrix", Computers and Mathematics with Applications, 61 (2011) 2596-2604.

[20] M. Razzaghi, Y. Ordokhani, "Solution of nonlinear Volterra Hammerstein integral equations via rationalized Haar functions", Math. Probl. Eng, 7 (2001) 205-219.

[21] F. Khellat, S. A. Yousefi, "The linear Legendre wavelets operational matrix of integration and its application", J. Franklin Inst, 343 (2006) 181-190.

[22] R. Burden, J.D. Faires, "Numerical Analysis, Fifth Edition”, PWS Publishing Company, Boston, (1993).

[23] W.H. Press, B.P. Flannery, S.A. Teukolsky, W.T. Vetterling, "Numerical Recipes", Cambridge University Press, Cambridge, (1988).

[24] E. Hairer, G. Wanner, "Stiff differential equations solved by Radau methods", J. Comput. Appl. Math, 111 (1999) 93-111.

[25] G. Sottas, "Rational Runge-Kutta methods are not suitable for stiff systems of ODEs", J. Comput. Appl. Math, 10 (1984) 169-174.

[26] D.L. Preiser, "A Class of nonlinear multistep A-stable numerical methods for solving stiff differential equations", J. Comput. Mathematics. Appl, 4 (1978) 43-51.

[27] Z. Jackiewicz, "Implementation of DIMSIMs for stiff differential systems", Applied Numerical Mathematics, 42 (2002) 251-267.

[28] J.L. Blue, H.K. Gummel, "Rational approximations to matrix exponential for systems of stiff differential equations", J. Compu. Phys, 51 (1970) 70-83.

[29] S.J. Liao, "Notes on the homotopy analysis method: some definitions and theorems", Common Nonlinear Sci Numer Simulat, 14 (2009) 91-97.

[30] J.H. He, "Variational iteration method for delay differential equations", Common. Nonlinear. Sci. Numer. Simul, 2 (1997) 235-236.

[31] J.H. He, "approximate solution of nonlinear differential equations with convolution product nonlinearities", Comput. Methods. Appl. Mech. Engrg, 167 (1998) 69-73.

[32] J.H. He, "Variational iteration method for autonomous ordinary differential systems", Appl. Math. Comput, 114 (2000) 115-123.

[33] N. Guzel, M. Bayram, "On the numerical solution of stiff systems", J. Appl. Math. And Comput, 170 (2005) 230-236. 